Nachtschicht im Altenheim

\section{Allein verantwortlich für 52 Bewohner}

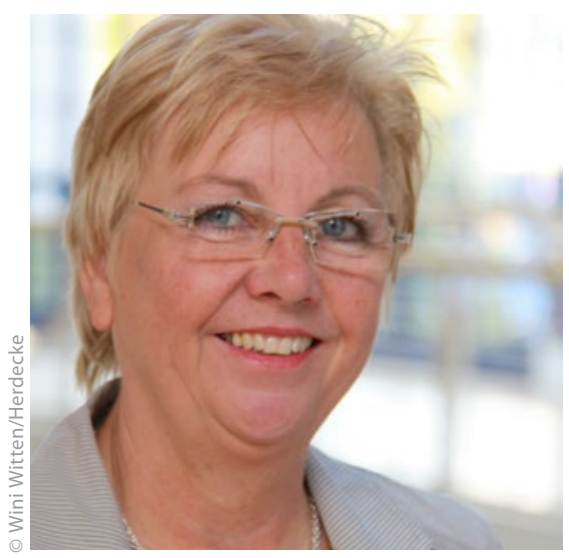

— In der bisher größten Studie zur Belastung von Pflegekräften in deutschen Altenheimen haben Pflegewissenschaftler der Universität Witten/Herdecke erschreckende Zahlen erhoben: Demnach ist eine einzelne Pflegekraft im Nachtdienst im Schnitt für 52 Personen zuständig. Und das meist ohne auf Entlastung durch einen Hintergrund- dienst zurückgreifen zu können. 8,7 Prozent der rund 280 Befragten gaben an, sogar für über 100 Personen zuständig zu sein. „Wer für 52 Personen in der Nacht zuständig ist, muss damit rechnen, dass hinter 26 Türen jederzeit jemand beim Weg zur Toilette stürzen kann", ordnet Studienleiterin Prof. Christel Bienstein die Ergebnisse ein. Rein rechnerisch blieben der Pflegekraft dann zwölf Minuten für jeden Patienten pro Nacht für Inkontinenzversorgung, Lagerung oder Verabreichung von Medikamenten. „Das ist Stress pur!“, macht Bienstein klar. Die Forscher haben die Rückmeldungen in einen Katalog münden lassen, in dem sie klare Forderungen zu Personalbesetzung und -qualifikation, Hintergrunddienst und Notfallleitlinien aufstellen. So müsse in der Nacht gewährleistet sein, dass mindestens zwei bis drei Pflegende für 60 Bewohner anwesend seien.

www.uni-wh.de

\section{Jahre dip \\ Forschung mit Profil}

- Das Deutsche Institut für angewandte Pflegeforschung e.V. (dip) feierte sein 15-jähriges Bestehen. Die Bilanz lässt sich sehen: Mehr als einhundert zum Teil umfangreiche Projekte im Volumen von insgesamt mehr als 11 Mio. Euro konnten umgesetzt werden. Bekannte Forschungslinien des Instituts sind z.B. das alle zwei Jahre erscheinende Pflege-Thermometer, zahlreiche Beiträge zur Pflegebildungsforschung sowie zur Pflegeprävention und -beratung. Aufträge und Projektförderungen fürs dip kommen von Ministerien in Bund und Ländern. Ebenso arbeiten seit Jahren auch Kranken- und Pflegekassen, Verbände, Stiftungen und Einrichtungsträger mit dem dip zusammen. So geht es in den Projekten beispielsweise um Demenz im Krankenhaus, um technische Assistenzsysteme im Alter oder um Curricula für innovative Qualifikationsansätze.

www.dip.de

\section{AOK Nordost \\ Pflegereport 2015}

- Angesichts zunehmender Pflegebedürftigkeit in der Bevölkerung sowie steigender Pflegezeiten der betroffenen Menschen kommt ambulanten Unterstützungs- und Entlastungsangeboten künftig eine Schlüsselrolle zu. Das ist eine Schlussfolgerung aus dem ersten Pflegereport der AOK Nordost, den das Gesundheitswissenschaftliche Institut Nordost (GeWINO) der Krankenkasse jetzt vorgelegt hat.Ein zentrales Ergebnis der ausgewerteten Abrechnungsdaten aller Pflegeleistungen von AOK Nordost-Versicherten aus den Jahren 2010-2014: 95\% der Pflegebedürftigen, die bereits Geldleistungen in Anspruch nehmen, bleiben im eigenen Zuhause, wenn sie sich zuvor haben beraten lassen. Gleichzeitig werden entlastende, ambulante Pflegeangebote zu selten genutzt.

Trotz Pflegebedürftigkeit wurden mehr als die Hälfte der Senioren bis an ihr Lebensende ausschließlich Zuhause umsorgt. Nur knapp 20 Prozent lebten in Pflegeheimen.

www.aok.de/nordost

Kirchliche Altenhilfeverbände Faire Entlohnung für gute Pflege

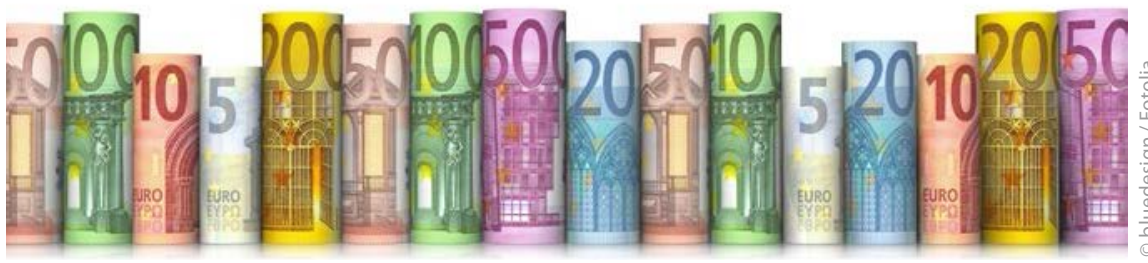

- Der Verband katholischer Altenhilfe in Deutschland e.V. (VKAD) und der Deutsche Evangelische Verband für Altenarbeit und Pflege (DEVAP) fordern für den Bereich der häuslichen Krankenpflege gemeinsam seit langem die Refinanzierung tarifvertraglicher Personalkosten in vollem Umfang durch die Krankenkassen. Die derzeit bestehende Gesetzeslücke in $\S 132$ a Absatz 2 SGB V nutzen die Krankenkassen aus und lehnen immer wieder eine volle Berücksichtigung gezahlter Tariflöhne bei den Vergütungen der häuslichen Krankenpflege ab. Dadurch entstehen den kirchlichen Sozialstationen wirtschaftliche Defizite mit den bekannten Folgen der immer größeren Arbeitsverdichtungen für die Mitarbeitenden. Auf diesen Missstand hatte schon die Petiti- on "Häusliche Krankenpflege - Tariflohn muss von den Krankenkassen vergütet werden" aufmerksam gemacht. Fast 60.000 Menschen schlossen sich innerhalb von nur vier Wochen der Forderung an und zeichneten die Petition.

VKAD und DEVAP appellieren an alle Akteure, sich für eine faire Entlohnung der Pflegefachkräfte in der ambulanten Pflege einzusetzen. Ihre qualifizierte und anspruchsvolle Arbeit muss angemessen tariflich bezahlt werden. Dazu werden bei den Finanzierungsgrundlagen gesetzliche Sicherheit und Klarheit benötigen. Diese Sicherheit wird geschaffen mit einer klaren Verankerung der Tariflohnanerkennung im Gesetz.

www.vkad.de und www.devap.info 